\title{
Zeros of eigenfunctions of some anharmonic oscillators
}

\author{
Alexandre Eremenko, Andrei Gabrielov and Boris Shapiro \\ July 28, 2018
}

\section{Introduction}

We consider eigenvalue problems of the form

$$
-y^{\prime \prime}+P(z) y=\lambda y, \quad y(-\infty)=y(\infty)=0
$$

where $P$ is a real even polynomial with positive leading coefficient, which is called a potential. The boundary condition is equivalent to $y \in L^{2}(\mathbf{R})$ in this case. It is well-known that the spectrum is discrete, and all eigenvalues $\lambda$ are real and simple, see, for example [3, 14]. The spectrum can be arranged in an increasing sequence $\lambda_{0}<\lambda_{1}<\ldots$

Eigenfunctions $y$ are real entire functions of order $(\operatorname{deg} P+2) / 2$ and each of them has finitely many real zeros. The number of real zeros of an eigenfunction is equal to the subscript of the corresponding eigenvalue $\lambda_{k}$. Asymptotic behavior of complex zeros of eigenfunctions is well-known, in particular, their arguments accumulate to finitely many directions, the socalled Stokes' directions [1, 2]. Using this one can show that for a real even potential $P$ of degree 4 with positive leading coefficient, all but finitely many zeros of each eigenfunction lie on the imaginary axis. See also [17] where a similar result was obtained for some cubic potentials.

Theorem 1. Let $P$ be a real even polynomial of degree 4 with positive leading coefficient. Then all non-real zeros of eigenfunctions y of the problem (11) belong to the imaginary axis.

\footnotetext{
*Supported by NSF grants DMS-0555279 and DMS-0244547.
} 
Under the assumptions of Theorem 1, every eigenfunction has infinitely many imaginary zeros.

For the special case that $P(z)=c z^{4}+d, c>0, d \in \mathbf{R}$ Theorem 1 was conjectured by Titchmarsh [16, p. 147] and proved by Hille [7, p. 617-618], see also [8, p. 188-190].

Operator considered in Theorem 1 is called a quartic anharmonic oscillator in quantum mechanics, and it was studied extensively by physicists and mathematicians. A very brief survey of known results is contained in Chapter I of [19].

For some potentials $P$, there exist eigenfunctions with finitely many zeros. Such eigenfunctions have the form

$$
y(z)=Q(z) \exp T(z)
$$

with polynomials $Q$ and $T$. For example, this is the case when $P$ is of degree 2 ; then all eigenfunctions are of the form (2), and $Q$ 's are the Hermite polynomials. Eigenfunctions with finitely many zeros can also occur for polynomials $P$ of higher degree, and these situations are of interest to physicists [9, 13, 18, 19]. It is easy to see that eigenfunctions of the form (2) can exist only in the case that $\operatorname{deg} P \equiv 2(\bmod 4)$. Moreover, for every $n$ such that $n \equiv 2(\bmod 4)$ there exist real polynomials $P$ such that some eigenfunctions have the form (2), see [5].

Theorem 2. Let $P$ be a real even polynomial of degree 6 with positive leading coefficient. If (2) is an eigenfunction of (11), then all non-real zeros of $Q$ belong to the imaginary axis.

Since the union of the real and imaginary axes does not contain any Stokes directions for a sextic potential $P$ (see Section 2), we conclude that eigenfunctions of a sextic potential with infinitely many zeros cannot have all zeros in the union of the real and imaginary axes.

In the proof of Theorem 2 we obtain a classification of eigenfunctions (2) which can occur in operators (11) with even sextic potential. It turns out that this classification fits the classification of the so-called "quasi-exactly solvable" sextic potentials [9, 19]. As a corollary we obtain in Section 6 that for even sextic potentials, eigenfunctions (2) can occur only for Lie-algebraic quasi-exactly solvable sextic potentials listed in [9, 19]. More precisely, Theorem 2 combined with the results of Turbiner and Ushveridze [19] gives the following 
Corollary. Let $P$ be a real even polynomial of degree 6 , and suppose that problem (11) has at least one solution y of the form (2). Then

$$
P(z)=c^{2} z^{6}+2 b c z^{4}+\left\{b^{2}-c(4 m+2 p+3)\right\} z^{2}+\text { const },
$$

where $c \in \mathbf{R} \backslash\{0\}, b \in \mathbf{R}, p \in\{0,1\}$ and $m$ a non-negative integer.

It was shown by Turbiner and Ushveridze that these potentials have exactly $m+1$ linearly independent eigenfunctions of the form (22). They correspond to the first $m+1$ even numbered eigenvalues if $p=0$ and to the first $m+1$ odd-numbered eigenvalues if $p=1$.

The proofs of theorems 1 and 2 are of purely topological nature, they are based on the study of the action of the symmetry group $\mathbf{Z}_{2} \times \mathbf{Z}_{2}$ of the problem (11) on certain partitions of the complex $z$-plane associated with the eigenfunctions.

Phenomenon described in Theorems 1 and 2 occurs only for potentials of degrees 4 and 6 :

Theorem 3. For every $k \geq 2$, there exists a real even polynomial $P$ of degree $4 k+2$ with positive leading coefficient, such that the problem (11) has an eigenfunction of the form (2), but the zero set of $Q$ is not a subset of the union of the real and imaginary axis.

Polynomial $P$ in Theorem 3 does not belong to the classification of quasiexactly solvable potentials that arise from finite-dimensional Lie algebras of differential operators in [9].

The third-named author is sincerely grateful to A. Turbiner for the hospitality at UNAM in October 2006 and inspiring discussions on the location of the roots of eigenfunctions of Schrödinger operators.

\section{Preliminaries}

From now on, we always assume that $P$ is real, even and has positive leading coefficient. We denote $d=\operatorname{deg} P$.

Making the change of the independent variable $z \mapsto-z$ we conclude that

every eigenfunction is either even or odd. We normalize even eigenfunctions by the condition

$$
y(0)=1,
$$


and the odd ones by the condition

$$
y^{\prime}(0)=1 \text {. }
$$

Consider another solution $y_{1}$ of the differential equation in (1) normalized by

$$
y_{1}(0)=0, \quad y_{1}^{\prime}(0)=1
$$

in the case that the eigenfunction $y$ is even, and

$$
y_{1}(0)=1, \quad y_{1}^{\prime}(0)=0
$$

in the case that the eigenfunction $y$ is odd. Then $y_{1}$ is even or odd, and its parity is opposite to the parity of $y$. Thus the meromorphic function $f=y / y_{1}$ is real and odd, in particular it is symmetric with respect to both real and imaginary axes: if we denote the reflections with respect to the coordinate axes by

$$
R(z)=\bar{z} \quad \text { and } \quad I(z)=-\bar{z}
$$

then

$$
f \circ R=R \circ f \quad \text { and } \quad f \circ I=I \circ f .
$$

The following facts are well-known [11, 12, 14]. The rays

$$
\rho_{j}=\{t \exp \{\pi i(2 j-1) /(d+2)\}: 0<t<\infty\}, \quad 0 \leq j \leq d+1
$$

are the Stokes' directions. They divide the plane into $d+2$ sectors $S_{j}$, where $S_{j}$ is bounded by $\rho_{j}$ and $\rho_{j+1}$. In each sector, each non-zero solution of the differential equation in (10) exponentially tends either to 0 or to $\infty$, (on every ray from the origin in this sector), in particular, $y(z) \rightarrow 0$ in $S_{0}$ and $S_{d / 2+1}$ in view of the boundary conditions in (1), while $y_{1}$ tends to $\infty$ in these two sectors.

Notice that the set of zeros and the set of poles of $f$ are both invariant with respect to $R$ and $I$.

Meromorphic function $f$ is of order $(d+2) / 2$, has no critical points (which means that $f^{\prime}(z) \neq 0$ and all poles are simple) and the set of its asymptotic values is finite. Such functions have been studied in great detail in [5, 6, 10, 11, 12, 14, 20]. If $A$ is the set of asymptotic values, then the restriction

$$
f: \mathbf{C} \backslash f^{-1}(A) \rightarrow \overline{\mathbf{C}} \backslash A
$$

is an (unramified) covering, and also $f$ is unramified at preimages of $A$. 
In each sector $S_{j}$, the function $f$ tends to an asymptotic value $a_{j}$ exponentially, and the asymptotic values in adjacent sectors are distinct. More precisely, for every sufficiently small $\epsilon>0$,

$$
f\left(r e^{i \theta}\right) \rightarrow a_{j}, \quad r \rightarrow \infty,
$$

uniformly with respect to $\theta \in\left[\rho_{j}+\epsilon, \rho_{j+1}-\epsilon\right]$. We have $a_{0}=a_{d / 2+1}=0$, and the symmetry properties (3) imply that $a_{j}=R\left(a_{-j}\right)$, and $a_{j}=I\left(a_{d / 2+1-j}\right)$. Here we understand the index $j$ as a residue modulo $d+2$.

The only singularities of the inverse function $f^{-1}$ are logarithmic branch points; they all lie over the asymptotic values. The total number of the logarithmic branch points is $d+2$, and they correspond to the $d+2$ sectors $S_{j}$.

As an example, consider the situation in Theorem 1 , where $d+2=6$. If we denote $a=a_{1}$ then the symmetry relations (3) imply that the asymptotic values are

$$
\left(a_{0}, a_{1}, a_{2}, a_{3}, a_{4}, a_{5}\right)=(0, a, I(a), 0,-a, R(a)) .
$$

Now, the condition that $a_{1} \neq a_{2}$ implies that $a$ cannot belong to the imaginary axis. We will later see in the course of the proof of Theorem 1 that $a$ cannot be real.

In Theorem 2, we have $d+2=8$ and the form of the eigenfunction (2) shows that $a_{2 k}=0, k=0,1,2,3$. Denoting $a_{1}=a$ again, we obtain from the symmetry relations (3) that

$$
\left(a_{1}, a_{3}, a_{5}, a_{7}\right)=(a, I(a),-a, R(a)) .
$$

We will see in the course of the proof of Theorem 2 that $a$ can be neither real nor pure imaginary. We conclude that in both theorems 1 and $2 f$ has five asymptotic values,

$$
a, I(a),-a, R(a) \text { and } \quad 0 .
$$

To study topological properties of the function $f$ one considers the pullback by $f$ of an appropriate cell decomposition of the Riemann sphere. The usual choice of this cell decomposition leads to an object which is called the line complex [4, 5, 6, 10, 11, 12, 20]. However classical line complexes are not convenient for our purposes because they do not reflect the symmetry relations (3), see, for example [5]. So in the main part of the proofs of theorems 1 and 2 (Sections 3 and 4) we use slightly different approach. 
However we find it more convenient to use the standard line complexes in the proof of Theorem 3. So we recall the definition of a line complex in the beginning of Section 5 .

\section{Common part of the proofs of Theorems 1 and 2}

In this Section, $f$ is a meromorphic function of finite order, with no critical points and five asymptotic values as in (4), where $a$ is neither real nor imaginary, and satisfies the symmetry conditions (3). We will treat the simpler case of real or imaginary a separately, in the end of Section 4.

We will work with partitions of a topological space $X$ which can be either the plane $\mathbf{C}$ or the Riemann sphere $\overline{\mathbf{C}}$ into subsets which we call vertices, edges and faces. All our partitions are locally finite, that is every point in $X$ has a neighborhood that intersects only finitely many edges, faces and vertices.

A vertex is just a point in $X$. An edge in $X$ is the image of the interval $(0,1)$ under a continuous map $\phi:[0,1] \rightarrow X$ whose restriction on $(0,1)$ is injective. The points $\phi(0)$ and $\phi(1)$ are the endpoints of the edge (they do not belong to the edge but always belong to $X$ ). The endpoints may be equal. We also say that the edge connects $\phi(0)$ with $\phi(1)$. The degree of a vertex $x$ of a partition is defined as the total number of ends of edges whose endpoints coincide with this vertex. Thus an edge with $x \in\{\phi(0), \phi(1)\}$, may contribute one or two units to the degree of $x$, one if $\phi(0) \neq \phi(1)$ and two if $\phi(0)=\phi(1)$. A face is a simply connected domain in $X$ whose boundary is locally connected.

A partition is a representation of $X$ as a locally finite disjoint union of faces, edges and vertices, such that all endpoints of all edges are vertices, and the boundary of every face consists of edges and vertices. We do not require that the closure of a face be homeomorphic to a closed disc.

We begin with the partition $C$ of the Riemann sphere $\overline{\mathbf{C}}$ which consists of:

- One vertex, $\infty$,

- Four edges $L_{k}, 1 \leq k \leq 4$, each beginning and ending at $\infty$, and such that $L_{k}$ separates one of the asymptotic values $a, I(a),-a, R(a)$ from all other asymptotic values. Moreover, we require that the union 
of these edges be invariant with respect to $R$ and $I$, more precisely, $L_{2}=I\left(L_{1}\right), L_{3}=-L_{1}$ and $L_{4}=R\left(L_{1}\right)$.

- Five faces $D_{k}, 0 \leq k \leq 4$ which are the components of $\mathbf{C} \backslash \cup_{k=1}^{4} L_{k}$. We enumerate them so that $0 \in D_{0}$, and $\partial D_{k}=L_{k} \cup\{\infty\}, 1 \leq k \leq 4$.

This partition is shown in Figure 1. In this illustration, $a$ belongs to the first quadrant.

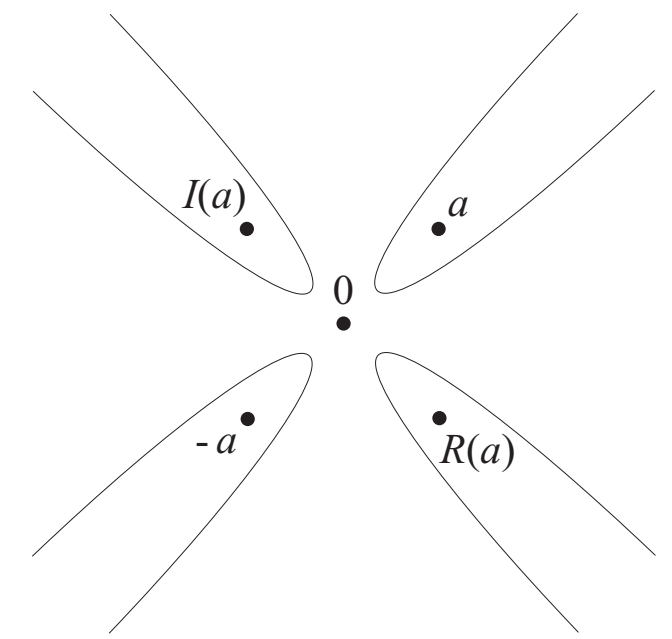

Fig. 1. Partition $C$ of the Riemann sphere.

Now we consider the preimage of $C$ under $f$. This is a partition $\Phi$ of the plane. Faces, edges and vertices of $\Phi$ are defined as components of the $f$-preimages of faces, edges and vertices of $C$.

So the vertices of $\Phi$ coincide with the poles of $f$. The degree of each vertex is 8 , the same as the degree of the vertex in the partition $C$.

The edges of $\Phi$ are disjoint curves connecting vertices. The edges can be of two types: an edge connecting a vertex to itself is called a loop, and an edge connecting two distinct vertices is called a simple edge.

We classify the faces of $\Phi$ into three types:

(i) Bounded faces whose boundaries consist of a loop and a vertex. We call them loop-faces. The closure of each loop-face is mapped by $f$ homeomorphically onto the closure of one of the $D_{k}$ with $1 \leq k \leq 4$.

(ii) Bounded faces which are mapped by $f$ homeomorphically onto $D_{0}$. (This homeomorphism does not necessarily extend to a homeomorphism between 
the closures!) Each of these faces contains exactly one zero of $f$, and each zero of $f$ belongs to one of these faces. We call them zero-faces.

(iii) Unbounded faces. The restriction of $f$ onto an unbounded face is a universal covering over $D_{k} \backslash b_{k}$ for some $k \in\{0, \ldots, 4\}$. Here $b_{k}$ is the asymptotic value which is contained in $D_{k}$. The boundary of each unbounded face consists of countably many edges and countably many vertices.

We label all faces by the corresponding asymptotic values: if a face is a component of the preimage of $D_{k}$ then its label is the asymptotic value $b_{k} \in D_{k}$. Bounded faces labeled by $b_{k}$ with $1 \leq k \leq 4$ are the loop-faces, while bounded faces labeled by 0 are the zero-faces.

We will need the following properties of the partition $\Phi$.

1. If two faces have a common boundary edge then their labels are distinct.

2. The 1-skeleton of $\Phi$ is connected. Indeed, the loops $L_{k}, 1 \leq k \leq 4$ generate the fundamental group of $\overline{\mathbf{C}} \backslash\left\{b_{0}, \ldots, b_{4}\right\}$. As

$$
f: \mathbf{C} \backslash f^{-1}\left(\left\{b_{0}, \ldots, b_{4}\right\}\right) \rightarrow \overline{\mathbf{C}} \backslash\left\{b_{0}, \ldots, b_{4}\right\}
$$

is a covering, we conclude that every pair of poles of $f$ can be connected by a curve which belongs to the 1-skeleton of $\Phi$.

3. Every edge connecting two different vertices belongs to the boundary of some unbounded face. Indeed, suppose that an edge $e$ connecting two different vertices belongs to the boundaries of two bounded faces $F_{1}$ and $F_{2}$. As the labels of these two faces are distinct (by property 1 above), one of these labels is not 0. But a bounded face whose label is not 0 has to be a loop-face (see (i)). So $e$ has to be a loop, which contradicts the assumption.

Now we transform our partition $\Phi$ into a tree. This is done in two steps.

Step 1. Remove all loop edges and all loop-faces. The resulting partition of the plane is called $\Phi^{\prime}$. Each face $F^{\prime}$ of $\Phi^{\prime}$ is a union of a face $F$ of $\Phi$ with some loops and loop-faces of $\Phi$. We label $F^{\prime}$ in $\Phi^{\prime}$ by the same label as $F$ had in $\Phi$. So all bounded faces of $\Phi^{\prime}$ are now labeled by 0 . They are Jordan regions with at least two boundary edges and at least two boundary vertices. It easily follows from the property 2 of $\Phi$ that $\Phi^{\prime}$ has connected 1-skeleton. Moreover, $\Phi^{\prime}$ is invariant with respect to both $I$ and $R$.

Step 2. Every bounded zero-face $F^{\prime}$ of $\Phi^{\prime}$ contains a unique zero of $f$. We call this zero an $\varnothing$-vertex and define a new partition $\Phi^{\prime \prime}$ of the plane in the 
following way. The vertices of the new partition are the vertices of $\Phi^{\prime}$, which we call now $\mathrm{x}$-vertices, and the new vertices which are called $\varnothing$-vertices. In other words, $\varnothing$-vertices are the zeros of $f$ and x-vertices are the poles of $f$. To define the edges of $\Phi^{\prime \prime}$ we connect each $\varnothing$-vertex in a zero-face $F^{\prime}$ of $\Phi^{\prime}$ to each x-vertex on the boundary of $F^{\prime}$ by a new edge inside $F^{\prime}$, so that these new edges are disjoint. This is possible to do because the closure of $F^{\prime}$ is locally connected. Then we remove all edges of $\Phi^{\prime}$ on the boundary of $F^{\prime}$. We perform this operation on every zero-face $F^{\prime}$ of $\Phi^{\prime}$.

Let us show that on Step 2, we can choose the new edges of $\Phi^{\prime \prime}$ in such a way that $\Phi^{\prime \prime}$ is symmetric with respect to both $R$ and $I$. Indeed, there are three possibilities for the orbit of a zero-face $F^{\prime}$ of $\Phi^{\prime}$ under the action of the group $\mathbf{Z}_{2} \times \mathbf{Z}_{2}$ generated by $I$ and $R$ : the orbit of $F^{\prime}$ can consist of one, two or four faces. We consider these possibilities separately.

If the orbit consists of 4 elements then $F^{\prime}$ is neither $R$-nor $I$-invariant. We choose the edges connecting the $\varnothing$-vertex in $F^{\prime}$ to the boundary x-vertices arbitrarily (with the only condition that they are disjoint), and then in other faces of the orbit of $F^{\prime}$ we use the images of these edges under the action of the group.

Suppose now that the orbit of $F^{\prime}$ consists of two elements, for example, $F^{\prime}$ is $R$-invariant but not $I$-invariant. We first define the new edges in $F^{\prime}$, so that the union of these new edges is $R$-invariant. This can be done if we notice that an $R$-invariant simply connected region intersects the real line by an interval. If some endpoint $x$ of this interval is a $\mathrm{x}$-vertex, we connect $x$ to $\varnothing$ by an interval of the real line. If $x \in \partial F^{\prime}$ is a $\mathrm{x}$-vertex in the upper half-plane, we connect it with $\varnothing$ by a curve in the intersection of $F^{\prime}$ with the upper half-plane, so that these curves for different x-vertices are disjoint. (We use here the fact that an intersection of a simply connected $R$-symmetric region with the upper half-plane is always connected, and its boundary is locally connected if the boundary of $F^{\prime}$ is.) Finally if $x \in \partial F^{\prime}$ is in the lower half-plane, we use the $R$-image of the edge connecting $\varnothing$ with $R(x)$. Then we define the new edges in the other face $I\left(F^{\prime}\right)$ of the orbit of $F^{\prime}$ as the $I$-images of the edges in $F^{\prime}$. The procedure for an $I$-invariant but not $R$-invariant face is the same. There are no other possibilities for an orbit of two elements: if $F^{\prime}=-F^{\prime}$ then $F^{\prime}$ has to be invariant with respect to the whole group, since $F^{\prime}$ is simply connected and a centrally symmetric simply connected region has to contain 0 .

The remaining case of $F^{\prime}$ which is both $R$ - and $I$ - invariant is treated 
similarly. The intersection of such face with the coordinate cross $\mathbf{R} \cup i \mathbf{R}$ consists of the union of two symmetric intervals, one on the real axis, another on the imaginary axis. To the $\mathrm{x}$-vertices at the endpoints of these intervals (if there are any such vertices) we draw straight edges from $\varnothing$. Then we notice that the intersection of $F^{\prime}$ with the first quadrant is connected and has locally connected closure. So we can draw the edges from $\varnothing$ to the $\mathrm{x}$-vertices in the first quadrant so that these edges are contained in the first quadrant. The remaining edges in $F^{\prime}$ are the images of those in the first quadrant under the symmetry group action.

The following Proposition summarizes the needed properties of $\Phi^{\prime \prime}$

Proposition 1. The partition $\Phi^{\prime \prime}$ has the following properties.

a) Its 1-skeleton is an infinite tree properly embedded in the plane.

b) Every edge belongs to the boundaries of two faces with distinct labels.

c) An ф-vertex cannot belong to the boundary of a face labeled 0.

d) Each $\mathrm{x}$-vertex is either connected with an $\varnothing$-vertex by an edge or belongs

to the boundary of a face labeled 0 .

e) $\Phi^{\prime \prime}$ has $d+2$ ends and $d+2$ faces.

f) Faces $F_{0}$ and $F_{d / 2+1}$ in a counter-clockwise order have labels 0 and these faces are interchanged by $I$.

Proof. First we prove that $\Phi^{\prime \prime}$ has no bounded faces. Suppose that $F^{\prime}$ is a bounded face of $\Phi^{\prime}$. It is a Jordan region with some number $k \geq 2$ boundary edges (see the description of Step 2). On Step 2 we replaced $F^{\prime}$ by certain number of triangles (=regions bounded by three edges), one triangle for each boundary edge of $F^{\prime}$, and then glued this triangle to some face $F_{0}^{\prime}$ of $\Phi^{\prime}$, exterior to $F^{\prime}$, along this edge. This face $F_{0}^{\prime}$ was unbounded by property 3 above. So it remains unbounded after adding triangles along some edges. So step 2 destroys all bounded faces and does not create new ones. Thus the 1-skeleton of $\Phi^{\prime \prime}$ is a forest. That it is connected, follows from connectedness of the 1-skeleton of $\Phi^{\prime}$; evidently Step 2 does not destroy connectedness.

It follows that the 1-skeleton of $\Phi^{\prime \prime}$ is a tree. As the vertices and edges accumulate only to infinity, the tree is properly embedded in $\mathbf{C}$. This proves a).

To prove b), we first notice that each edge of $\Phi^{\prime \prime}$ that comes from $\Phi^{\prime}$ belongs to the boundaries of two faces with distinct labels (property 1 above). So it remains to prove b) for the new edges added on Step 2. Let $e$ be an edge added on step 2. It connects an $\varnothing$ and $\mathrm{x}$ inside a zero-face $F^{\prime}$ of $\Phi^{\prime}$. Let $e_{1}$ and $e_{2}$ be the two edges on the boundary of $F^{\prime}$ incident to the common 
vertex $\mathrm{x}$. Then $e_{1}$ and $e_{2}$ are $f$-preimages of two different edges $L_{k}$ and $L_{j}$ of $C$, and thus the faces $F_{1}^{\prime}$ and $F_{2}^{\prime}$ exterior to $F^{\prime}$ that have $e_{1}$ and $e_{2}$ on their boundaries have distinct labels. Thus $e$ is a common boundary edge of two faces with distinct labels. This proves $b$ ).

To prove c), we consider an $\varnothing$-vertex and the bounded face $F^{\prime}$ of $\Phi^{\prime}$ which contains this vertex. This face $F^{\prime}$ is labeled by 0 (as all bounded faces of $\Phi^{\prime}$ are), and thus there cannot be an edge in $\Phi^{\prime}$ in the common boundary of $F^{\prime}$ and another face labeled by 0 . It follows that Step 2 cannot produce an $\varnothing$-vertex on the boundary of a face with label 0 .

To show d), we first notice that the number of unbounded faces and the labeling of these faces do not change when we perform steps 1 and 2. Now statement e), as well as $\mathrm{f}$ ) follow from the asymptotic properties of $f$ stated in the Preliminaries: each unbounded face (of any partition $\Phi, \Phi^{\prime}$ or $\Phi^{\prime \prime}$ ) is asymptotic to one of the sectors $S_{j}$ where $f(z)$ tends to the label of this face. The total number of sectors is $d+2$, and the bisectors of two of them are the positive and negative rays of the real line.

This completes the proof of Proposition 1.

In the next Section we will classify all trees satisfying the conclusions of the Proposition 1 with $d=4$. For $d=6$ we will use an additional property that arises from (2): that every other face of $\Phi^{\prime \prime}$ is labeled by 0 .

It will result from this classification that all trees arising from the eigenfunctions in theorems 1 and 2 as 1-skeletons of $\Phi^{\prime \prime}$ have the following property: each $\varnothing$-vertex is either fixed by $R$ or fixed by $I$. As the $\varnothing$-vertices are the zeros of $f$ and thus the zeros of the eigenfunction $y$, this will prove our theorems 1 and 2.

\section{Completion of the proofs of theorems 1 and 2}

We classify the embedded trees up to homeomorphisms of the plane that commute with both $R$ and $I$, send vertices to vertices and preserve the labels of faces and vertices ( $\varnothing$ and $\mathrm{x}$ ).

Proposition 2. For $d=4$, there are only three types of embedded trees satisfying the conclusions of Proposition 1; they are shown in Fig. 2. All $\varnothing$-vertices lie in the union of the real and imaginary lines. All trees of these three types are parametrized by a non-negative integer $n$ : the number of $\varnothing$ vertices on the real line. First type occurs when $n=0$, second for even $n \geq 2$ and third when $n$ is odd. 
1)

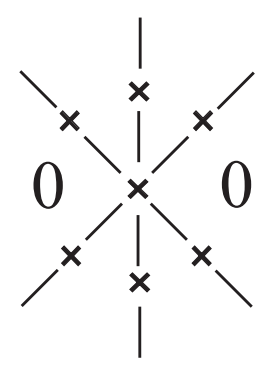

2)

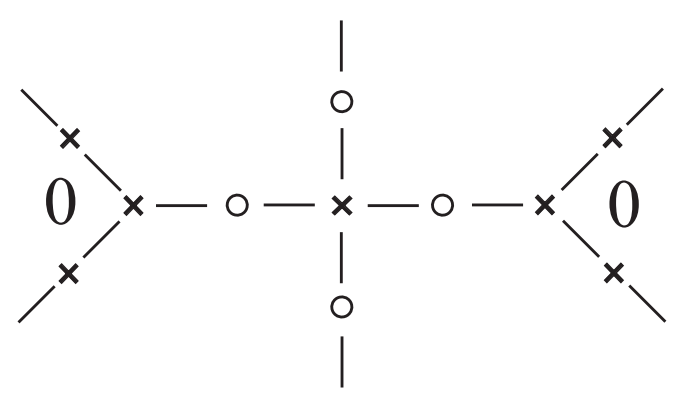

3)

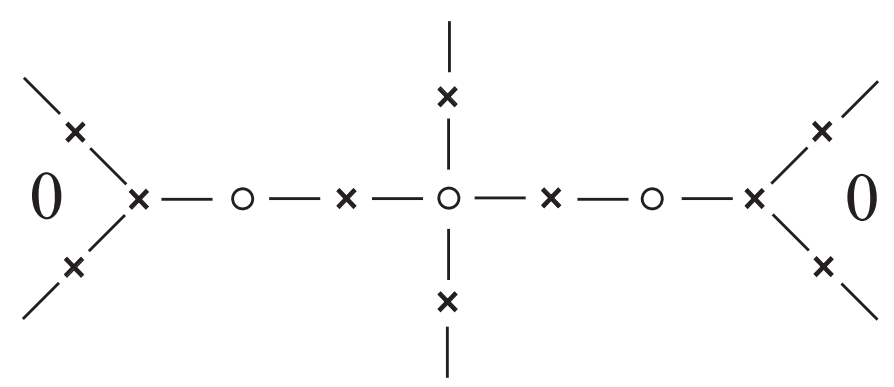

Fig. 2. Types of possible trees in Theorem 1.

Proof. If vertices of order 2 are ignored, there are two topological types of properly embedded trees with 6 ends, both $I$ and $R$ symmetries and satisfying b) and f) of Proposition 1:

a)

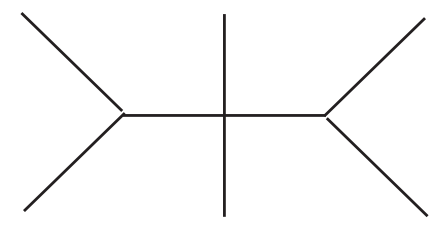

b)

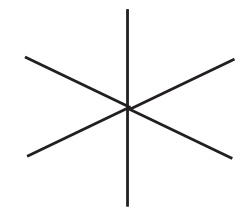

Fig. 3. Topological types of possible trees in Theorem 1 (ignoring vertices of order 2).

This simple fact can be proved along the same lines as Proposition A in the Appendix and it is left to the reader. Now statements c) and d) of Proposition 1 imply that the $\varnothing$-vertices lie on the coordinate cross.

For Theorem 2, we have one property in addition to those stated in Proposition 1: every even-numbered face (in the natural cyclic order) is labeled by 0 . 
Proposition 3. For $d=6$, there are only five types of embedded trees satisfying the conclusions of Proposition 1 and the additional property that every even-numbered face is labeled by 0 . They are shown in Figure 4. These trees are parametrized by two integers: the total number $m$ of $\varnothing$-vertices and the number $n$ of $\varnothing$-vertices on the real line. These integers satisfy the following evident restrictions: $0 \leq n \leq m$ and $n-m$ is even.

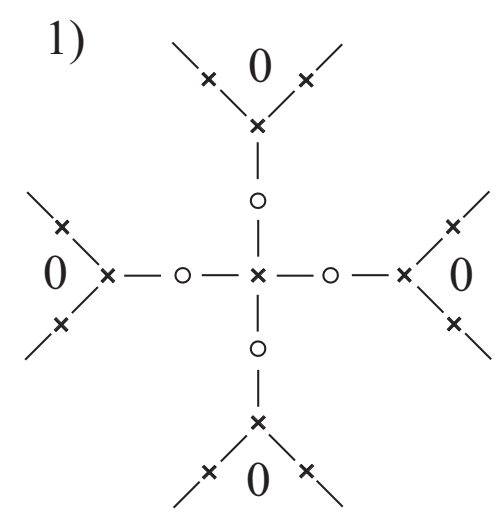

3)

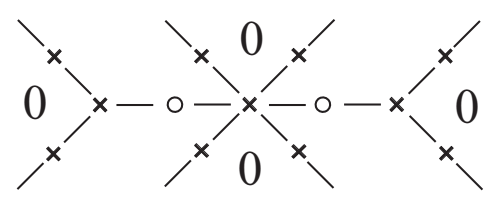

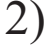

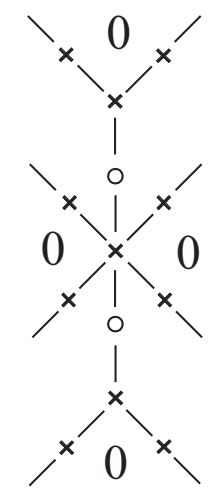

4)

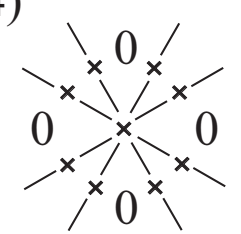

5)

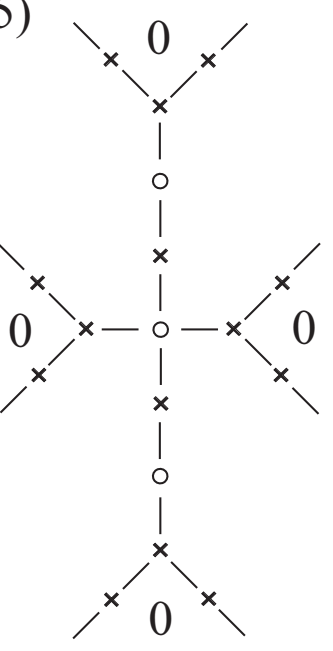

Fig. 4. Possible types of trees in Theorem 2.

Proof. Only topological types a), c), f) and j) of Proposition A in the Appendix satisfy conditions b) and f) of Proposition 1. Topological type a) gives two types of trees, depending on the type of vertex in the center.

One can show, using a result of Nevanlinna cited in the next Section that all types of trees described in propositions 2 and 3 can actually occur for the eigenfunctions in theorems 1 and 2.

To complete the proof of Theorem 1, it remains to prove that $a$ cannot be real. The proof is by contradiction. If $a$ is real, we have only three asymptotic values, $0, a,-a$. We repeat with simplifications the construction in Section 3. The partition $C$ of the Riemann sphere has two edges and three faces now: 


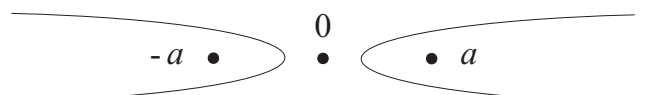

Fig. 5. Partition $C$ in the case of real $a$.

The degree of each vertex of $\Phi$ is 4 . The boundary of each zero-face in $\Phi$ contains two edges and two vertices, and thus it contains no loops. Proposition 1 remains true for the partition $\Phi^{\prime \prime}$. But now an analog of the Propositions 2 and 3 shows that no admissible graphs exist. Indeed, graphs of the types 2 and 3 in Fig. 2 are excluded because two faces labeled $a$ have common edges in them. Graph of the type 1 is excluded because it has a vertex of degree 6. For the same reasons, all graphs in Fig. 4 are excluded. Thus a cannot be real.

To complete the proof of Theorem 2, we have to show that $a$ can be neither real nor imaginary. The argument is the same as we just used to show that $a$ cannot be real in Theorem 1 , and it is left to the reader.

\section{Theorem 3}

We begin by recalling the definition of a line complex. Let $f$ be a meromorphic function of finite order without critical points and with finitely many asymptotic values.

Let $\Gamma$ be an oriented Jordan curve in the Riemann sphere $\overline{\mathbf{C}}$, which passes once through each asymptotic value. Orientation of $\Gamma$ induces a cyclic order on the set of asymptotic values. We denote the asymptotic values in this order by $\left(b_{1}, \ldots, b_{q}\right)$. The subscripts $1, \ldots, q$ in $b_{k}$ are considered as residues modulo $q$, so that $b_{q+1}=b_{1}$, etc. The curve $\Gamma$ with marked points $b_{k}$ is called the base curve, and the points $b_{k}$ are called the base points.

Choose one point $\varnothing$ inside $\Gamma$ and one point $\mathrm{x}$ outside $\Gamma$. Then connect $\varnothing$ and $\mathrm{x}$ by $q$ disjoint edges $\Gamma_{1}, \ldots, \Gamma_{q}$, so that each $\Gamma_{k}$ intersects $\Gamma$ exactly once, and this intersection happens on the open $\operatorname{arc}\left(b_{k}, b_{k+1}\right)$ of $\Gamma$.

The points $\varnothing$ and $\mathrm{x}$, the curves $\Gamma_{k}$ and the components of the complement to the union of these curves and points form a partition of the Riemann sphere with two vertices, $q$ edges and $q$ faces.

The $f$-preimage of this partition of the plane $\mathbf{C}$ is traditionally called the line complex $L$. The faces, edges and vertices of the line complex can be naturally labeled by the names of their images. A line complex is considered 
as a topological object: two line complexes are equivalent if they can be mapped one onto another by an orientation preserving homeomorphism of the plane.

The following properties of a line complex are known [4, 6, 10, 11, 12 , and easy to prove.

The 1-skeleton of $L$ is a bi-partite graph (in particular it has no loops). Every vertex belongs to the boundaries of exactly $q$ faces, and the labels of these $q$ faces are all distinct; the labels $\left(a_{1}, \ldots, a_{q}\right)$ of these faces follow anti-clockwise around an $\varnothing$-vertex and clockwise around a $\mathrm{x}$-vertex.

The faces are of two types: 2-gons and $\infty$-gons. The 2-gons are mapped by $f$ homeomorphically onto neighborhoods of the base points. So each $2-$ gon contains exactly one simple solution of the equation $f(z)=b_{k}$, where $b_{k}$ is the label of this 2-gon. $\infty$-gons correspond to the logarithmic branch points of $f^{-1}$, and the restriction of $f$ onto an $\infty$-gon is a universal cover of a punctured neighborhood of the base point $b_{k}$, where $b_{k}$ is the label of this $\infty$-gon. In particular, the equation $f(z)=b_{k}$ has no solutions in an $\infty$-gon labeled $b_{k}$.

An unlabeled line complex can be defined intrinsically, without any reference to $f$. It is a partition of the plane into vertices, edges and faces with the following property:

The 1-skeleton is a connected bi-partite properly embedded graph whose all vertices have the same degree $q$.

If such partition of the plane is given, there are two choices of labeling the vertices with $\mathrm{x}$ and $\varnothing$. Furthermore, if a cyclically ordered set of points $\left(b_{1}, \ldots, b_{q}\right)$ is given, we can always label the faces of our partition with elements of this set, so that the subscripts of the labels increase counterclockwise around each $\varnothing$-vertex and clockwise around a x-vertex. Notice that such labeling is uniquely defined once the label of one face is specified, and the label of one face can be prescribed arbitrarily.

A fundamental theorem which is due to Nevanlinna [11], see also [6], says the following.

Suppose that a labeled line complex is given with $d+2$ unbounded faces and all bounded faces are 2-gons. Choose a base curve passing through the labels according to their cyclic order. Then there exists a meromorphic function $f$ in the plane of order $(d+1) / 2$ with no critical points and whose line complex with respect to the this base curve is equivalent to the given one. 
This function is unique up to a change of the independent variable $z \mapsto$ $c z+b, c \neq 0$.

Each $f$ given by this theorem is a ratio of two linearly independent solutions of the differential equation $-y^{\prime \prime}+P y=0$ where

$$
-2 P=\frac{f^{\prime \prime \prime}}{f^{\prime}}-\frac{3}{2}\left(\frac{f^{\prime \prime}}{f^{\prime}}\right)^{2} .
$$

We use this result to prove Theorem 3. For simplicity of illustrations, we consider only the case $d=10$. Then we should have 12 sectors $S_{j}$, and we choose the asymptotic values in these sectors to be $a_{0}=a_{2}=a_{4}=a_{6}=a_{8}=$ $a_{10}=0$ and

$$
\left(a_{1}, a_{3}, a_{5}, a_{7}, a_{9}, a_{11}\right)=(a, b, a, R(a), R(b), R(a)),
$$

where $a$ and $b$ are distinct and belong to the positive imaginary axis, for example one can take $a=2 i$ and $b=i$ as we do in our pictures. So we have 5 asymptotic values. Then we choose the imaginary axis (oriented "up") as our base curve, the $\varnothing$ point at -1 and the $\mathrm{x}$ at +1 .

Now we consider the following line complex: 

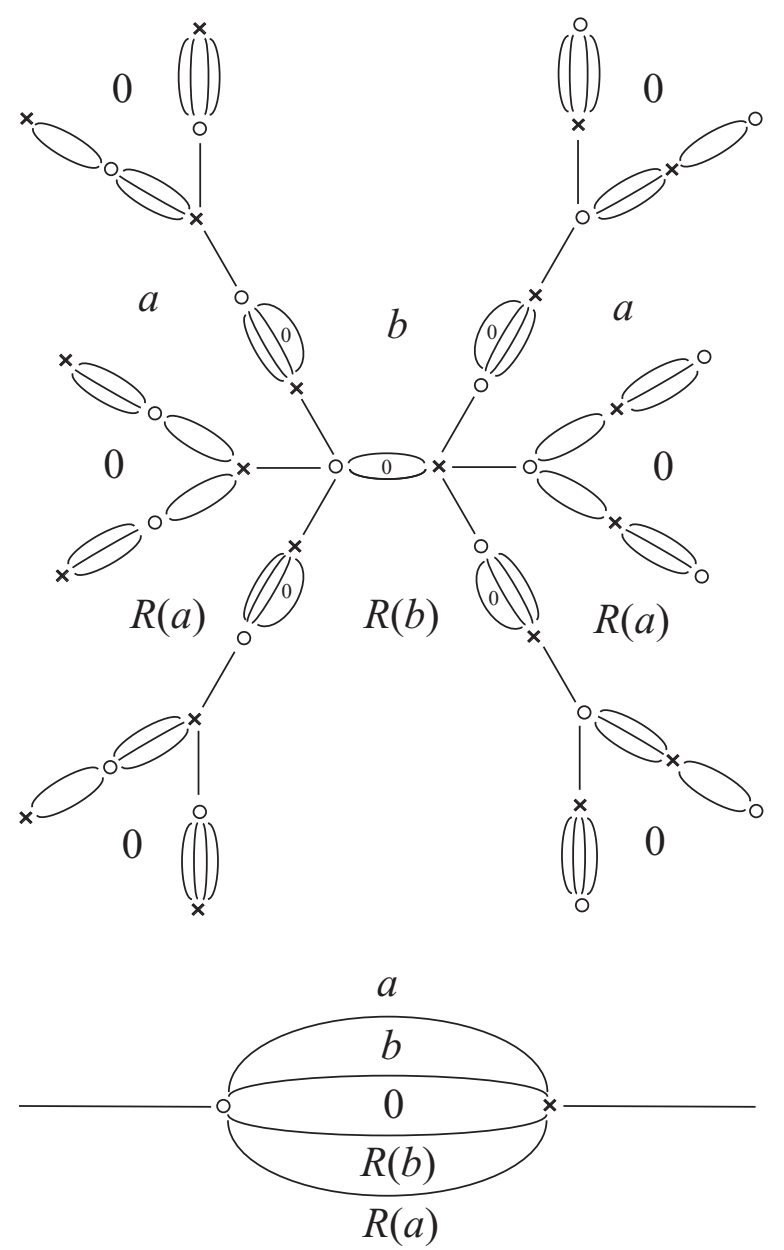

Fig. 6. Line complex for Theorem 3. Curves $\Gamma_{k}$ are shown in the bottom.

Meromorphic function $f$ corresponding to this complex by Nevanlinna's theorem will be normalized as follows: $\varnothing^{*}=-1$ and $\mathrm{x}^{*}=1$, where $\varnothing^{*}$ and $\mathrm{x}^{*}$ are the two vertices closest to the center of the picture. Then it is clear that $f$ has the necessary symmetry properties (3). Four zeros shown in the picture form a single orbit under the $\mathbf{Z}_{2} \times \mathbf{Z}_{2}$ action. So these zeros are neither real nor imaginary.

\section{Quasi-exactly solvable sextic potentials}

Here we prove the Corollary stated in the Introduction. 
In 1987, Turbiner and Ushveridze (see, a detailed exposition in [19]) discovered the family of sextic potentials

$$
P_{m, p, b}(z)=z^{6}+2 b z^{4}+\left(b^{2}-4 m-2 p-3\right) z^{2},
$$

where $m$ is a non-negative integer, $p \in\{0,1\}$, and $b \in \mathbf{R}$, with the following remarkable property. Problem (1) with $P=P_{m, p, b}$ has $m+1$ eigenfunctions of the form

$$
y(z)=z^{p} Q_{k, m, p, b}\left(z^{2}\right) \exp \left(\frac{z^{4}}{4}-b \frac{z^{2}}{2}\right), \quad 0 \leq k \leq m
$$

where $Q_{k, m, p, b}$ are polynomials of degree $m$. These eigenfunctions correspond to the eigenvalues $\lambda_{2 k+p}$ of the Schrödinger operators (11) with $P=P_{m, p, b}$. Such potentials, having several first eigenfunctions of the form (2), are sometimes called quasi-exactly solvable [19].

Using the method which goes back to Stieltjes [15], Ushveridze proved that all zeros of the polynomials $Q_{k, m, p, b}$ are real [19], pp. 53-56, so all zeros of the eigenfunctions belong to the union of the real and imaginary axes. More precisely, $Q_{k, m, p, b}$ has $k$ positive and $m-k$ negative zeros.

On the other hand, in the proof of our Theorem 2 we obtained the classification of all meromorphic functions $f=f_{k, n, p, a}$ arising from eigenfunctions of the form (2) for all problems (11) with even polynomials $P$ of degree 6 . Here $f_{k, n, p, a}$ is the function $f$ from Section 2 with $2 n+p$ zeros, $2 k+p$ of them real, and asymptotic value $a$ in the first quadrant.

Proportional functions $f$ belong to the same potential $P$ and the same eigenvalue, hence we can normalize so that $a=\exp (i \alpha)$ where $\alpha \in(0, \pi / 2)$ when $p=1$ and $\alpha \in(-\pi / 2,0)$ when $p=0$.

Thus we obtain a family of functions $\alpha=g_{k, m, p}(b)$. To each potential $P_{m, p, b}$ and each $k \in[0, m]$ this function $g$ puts into correspondence the argument of the asymptotic value $a$ of the corresponding function $f=y / y_{1}$, where $y$ is the $2 k+p$-th eigenfunction of (11), and $y_{1}$ is a second linearly independent solution of the differential equation in (1), with $P=P_{m, p, b}$ and $\lambda=\lambda_{2 k+p}$. This function $f$ is $f_{k, m, p, a}$ for some $a$, and we define $g_{k, m, p}(b)=\operatorname{Arg} a$. Moreover, $f$ is normalized so that $|a|=1$ and $0<|\operatorname{Arg} a|<\pi / 2$.

Thus each function $g$ maps the real line into an open interval $J \in \mathbf{R}$, where $J=(0, \pi / 2)$ for $p=1$, and $J=(-\pi / 2,0)$ for $p=0$. We have to prove that all these functions $g$ are surjective.

It is well-known that $g$ is continuous and real analytic (see, for example, [14]). On the other hand, for each $a \in J$, there is a neighborhood $V \subset J$ of 
$a$, such that a (right) inverse branch $h: J \rightarrow \mathbf{R}$ of $g$ can be defined, that is $h \circ \phi=\mathrm{id}_{J}$, and moreover, this $h$ is real analytic [14]. We conclude that $g$ is surjective and this proves the Corollary.

\section{Appendix. Classification of trees}

In this Appendix we classify all trees with 8 ends, that have no ends on the real and imaginary axes, and are both $R$ - and $I$-symmetric. In what follows we call such trees double-symmetric. We say that two double-symmetric trees are isomorphic if there exists an orientation-preserving and commuting with both $R$ and $I$ homeomorphism of the complex plane sending one to the other. We do not require that this homeomorphism sends vertices to vertices.

Proposition A There exist 11 non-isomorphic double-symmetric trees with no ends fixed by either $R$ or $I$, see Figure $\%$.

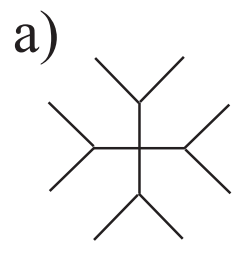

a) b)

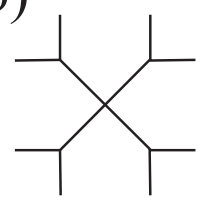

c)

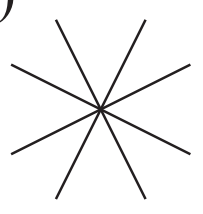

d)

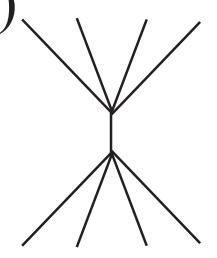

e)

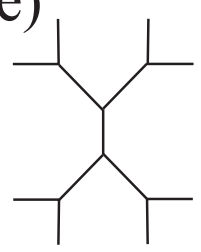

f)

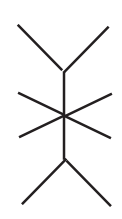

g)

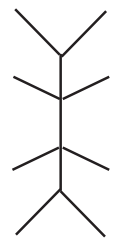

h)

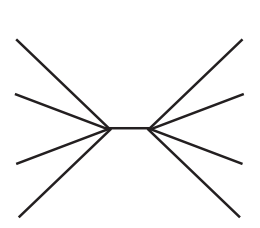

i)

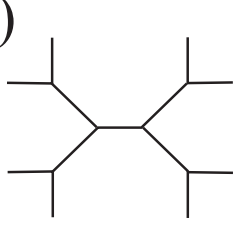

j)

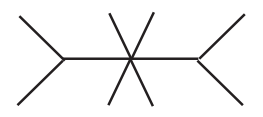

$\mathrm{k})$

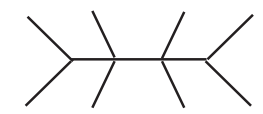

Fig. 7. Double symmetric trees with 8 ends none of which is on the axes.

The proof uses the following simple lemma classifying symmetric planar rooted trees with 4 ends whose proof is left to the reader. 
Lemma. There exist 6 non-isomorphic planar rooted trees with 4 ends (not counting the root) and no vertices of degree 2 , symmetric with respect to a reflection in a line passing through the root, see Figure 8.

a)

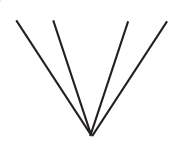

d)

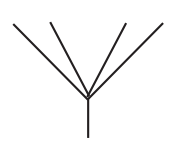

b)

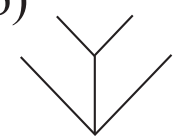

e)

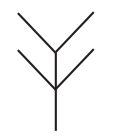

c)

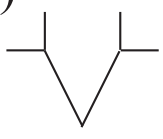

f)

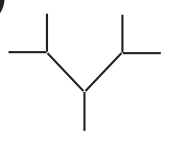

Fig. 8. I-symmetric rooted trees with 4 ends.

To prove Proposition A we argue as follows. Any double-symmetric tree contains the origin (since a doubly symmetric 1-complex embedded in the plane and not containing the origin either is either disconnected or contains a cycle). Consider the intersection of such a tree with the coordinate axes. If it intersects both axes non-trivially, i.e., not only at the origin, then the only possibility is the tree marked $a$ in Figure 7 . Assume now that a doublesymmetric tree intersects the real axis only at the origin. Then the intersection of that tree with the closed upper half-plane is a planar tree rooted at the origin with 4 ends, symmetric with respect to $I$. By the Lemma, there are exactly 6 such trees. They correspond to the trees marked $b$ through $g$ in Figure 7. To get all the remaining double-symmetric trees we have to rotate the latter 6 trees by $\pi / 2$. Notice that trees marked $b$ and $c$ are invariant under this rotation, producing just 4 new trees, namely, trees marked $h$ through $k$ in Figure 7. Thus the total number of trees equals $1+6+4=11$.

\section{References}

[1] Bank, S., A note on the zeros of solutions $w "+P(z) w=0$ where $P$ is a polynomial, Appl. Anal. 25 (1987), no. 1-2, 29-41.

[2] Bank, S., Addendum to: "A note on the zeros of solutions of $w$ " $+P(z) w=$ 0 where $P$ is a polynomial", Appl. Anal. 25 (1987), no. 1-2, 29-41.

[3] F. A. Berezin and M. A. Shubin, The Schrödinger equation, Kluwer, Dordrecht, 1991. 
[4] E. Drape, Über die Darstellung Riemannscher Flächen durch Streckenkomplexe, Deutsche Math., 1 (1936) 805-824.

[5] A. Eremenko and S. Merenkov, Nevanlinna functions with real zeros, Illinois J. Math., 49, 3-4 (2005) 1093-1110

[6] A. A. Goldberg and I. V. Ostrovskii, Distribution of values of meromorphic functions, Moscow, Nauka, 1970 (English translation to appear in AMS).

[7] E. Hille, Lectures on ordinary differential equations, Addison-Wesley, Menlo Park, CA, 1969.

[8] E. Hille, Ordinary differential equations in the complex domain, John Wiley and sons, NY, 1976.

[9] A. González-Lopéz, N. Kamran and P. Olver, Normalizability of onedimensional quasi-exactly solvable Schrödinger operators, Comm. Math. Phys. 153 (1993) 117-146.

[10] R. Nevanlinna, Über die Herstellung transzendenter Funktionen als Grenzwerte rationaler Funktionen, Acta Math. 55, (1930) 259-276

[11] R. Nevanlinna, Über Riemannsche Flächen mit endlich vielen Windungspunkten, Acta Math. 58, 295-373 (1932).

[12] R. Nevanlinna, Eindeutige analytische Funktionen. 2-te Aufl. BerlinGöttingen-Heidelberg, 1953.

[13] M. Shifman, Quasi-exactly-solvable spectral problems and conformal field theory, in the book: N. Kamran and P. Olver, eds., Lee algebras, cohomology and new applications in quantum mechanics, Contemp. Math., 160, AMS, Providence, RI, 1994.

[14] Y. Sibuya, Global theory of a second order linear ordinary differential equation with a polynomial coefficient, North-Holland Publishing Co., Amsterdam-Oxford; American Elsevier Publishing Co., Inc., New York, 1975 .

[15] T. Stieltjes, Sur certains polynômes qui vérifient une équation differentielle linéaire du second ordre et sur la théorie des fonctions de Lamé, 
Acta Math. 6 (1885) 321-326; Oeuvres complétes, Vol. 1, Springer, Berlin, 1993, 434-439.

[16] E. Titchmarsh, Eigenfunction expansions associated with second order differential equations, Clarendon press, Oxford, 1946.

[17] Duc Tai Trinh, Asymptotique et analyse spectrale de l'oscillateur cubique, Thése, Université de Nice, 2002.

[18] A. Turbiner, Quasi-exactly-solvable problems and sl(2) algebra, Comm. Math. Phys., 118 (1988) 467-474.

[19] A. Ushveridze, Quasi-exactly solvable models in quantum mechanics, Inst. of Physics Publ., Bristol, 1994.

[20] H. Wagner, Über eine Klasse Riemannscher Flächen mit endlich vielen nur logarithmischen Windungspunkten, J. für Math., 175, 1 (1936) 6-49.

Purdue University

West Lafayette, IN

47907-2067 U.S.A.

eremenko@math.purdue.edu

agabriel@math.purdue.edu

Stockholm University

Stockholm, S-10691, Sweden

shapiro@math.su.se 